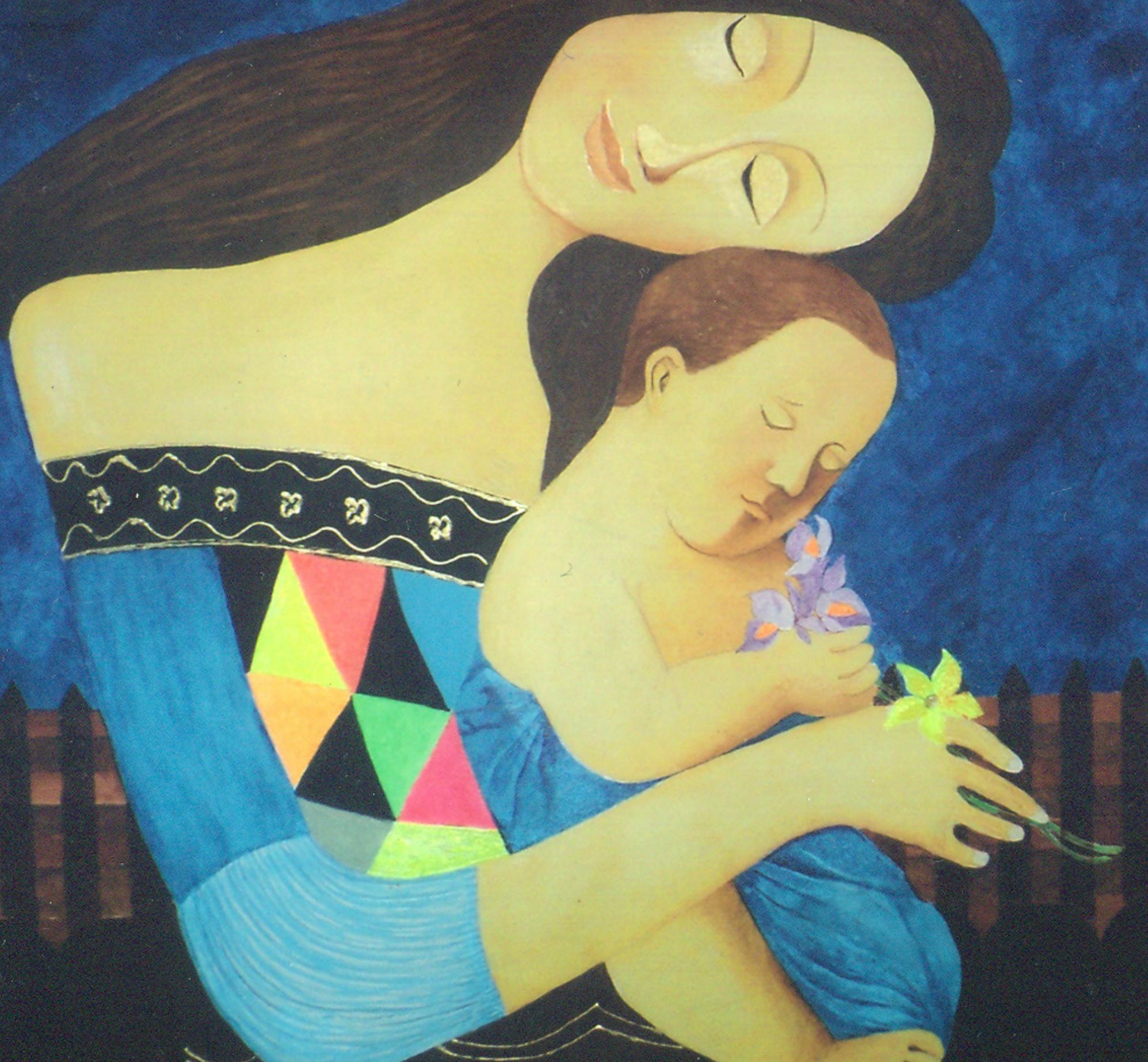




\title{
2 La migración de retorno de migrantes mexicanos en el siglo XXI
}

\author{
Ofelia Woo Morales*, Alma leticia Flores Ávila**
}

RESUMEN. La migración de mexicanos hacia Estados Unidos es añeja así como el retorno de los migrantes; sin embargo, es de reciente atención el estudio de lo que se considera el migrante retornado contemporáneo debido al volumen de la migración de retorno que aumentó considerablemente después de 2007, atribuido a la crisis económica de Estados Unidos y las políticas migratorias de ese país, que son cada vez más restrictivas. Se hace referencia por varios autores que el migrante que retorna en la actualidad es diferente que el de antaño. Por lo que interesa presentar en este artículo, con diversas escalas de análisis (nacional, regional, estatal, localidad), a dónde está llegando el migrante retornado y sus características sociodemográficas de acuerdo a la información del Censo de Población y Vivienda de 2010.

Palabras Clave: Proceso migratorio, Migración de retorno, perfil del migrante retornado.

\begin{abstract}
The migration of Mexicans to the United States is as old as the return of migrants, however, it is recent the study of what has been considered the contemporary returned migrant since the significantly increased of the volume after 2007; this is attributed to the economic crisis in the United States and the recent immigration policies which are more restrictive. It is referred by several authors that the current return migrant is different than the olden days, for what is very important to us introduce this article with multiple scales analysis (national, regional, state and by location) where the return migrant is coming and their sociodemographic characteristics according to information from the Census of Population and Housing 2010.
\end{abstract}

Keywords: Migration process, return migration, returning migrant profile.

\section{Introducción}

México es un país de origen, tránsito, destino y retorno de migrantes. El proceso de migración de mexicanos hacia Estados Unidos tiene más de cien años y ha tenido importantes transformaciones a lo largo de ese tiempo, tanto en el perfil del migrante como en las características del patrón migratorio. De ser una migración predominantemente masculina, temporal, relacionada al mercado laboral agrícola de Estados Unidos, se transformó en una migración de mayor permanencia, motivada por factores económicos, sociales y culturales; ya no sólo emigran los hombres de áreas rurales para trabajar los campos agrícolas de Estados Unidos, también proceden de áreas urbanas incorporándose principalmente al sector de servicios y construcción. Se ha registrado la emigración de mujeres y jóvenes, que lo hacen por razones laborales y/o proyectos individuales o familiares (Durand y Massey 2003, Arias y Woo 2004, Flores 2010, Castañeda 2014).

En este artículo interesa destacar la heterogeneidad en las características de la población de retorno en el siglo XXI, al considerar migrante de retorno contemporáneo lo señalado por Rivera (2011), que de acuerdo a esa autora el proceso de retorno está relacionado a factores estructurales, dinámicas de mercados laborales internacionales y nacionales, así como las condiciones de los lugares de origen y destino.

El migrante de retorno contemporáneo en México es diferente al que se había identificado en el siglo XX, predominantemente masculino. Su regreso estaba ca-

Recibido en mayo 2015 / Aceptado y versión final junio 2015

* Licenciada en Sociología, Maestría en Ciencias Sociales, Doctorado en Ciencias Sociales, Miembro del Sistema Nacional de Investigadores de México Profesora-Investigadora del Departamento de Estudios Socio Urbanos, e-mail ofewoo@yahoo.com

** Licenciada en Trabajo Social, Maestra en Ciencias Sociales, Doctora en Ciencias Sociales. Miembro del Sistema Nacional de Investigadores de México. Profesora Investigadora Titular en la Universidad de Guadalajara, e-mail aleticia2003@yahoo.com.mx 
racterizado por las condiciones de oferta y demanda de mano de obra, o inclusive al éxito o fracaso del llamado sueño americano.

México ha registrado un importante aumento en la migración de retorno desde el 2000 a la fecha. Asociada a las crisis económica (2001 y 2008) y a las políticas migratorias de Estados Unidos que cada vez son más restrictivas.

Como ha sido ampliamente señalado por diversos autores, la migración de retorno está relacionada con el proceso de emigración; no es el acto de regresar, es una etapa más de la trayectoria del migrante (Cassarino 2004, Rivera 2011): "El retorno es parte de la dinámica de la emigración, es un elemento constitutivo que está presente en el lugar de origen y destino, una etapa más del ciclo migratorio" (Cassarino 2004, pag16).

La población que ha retornado a principios de este siglo tiene características diferentes. Es una población más heterogénea, regresan hombres, mujeres, jóvenes, niños, familias (Escobar 2012, López y Mojica 2013). De ahí el interés en conocer cuáles son las características sociodemográficas e identificar si existe una relación entre los lugares de origen y el retorno.

El artículo se estructura en tres apartados, se presenta de manera sucinta la revisión bibliográfica que da cuenta de la migración de retorno en diversas latitudes y en México; posteriormente está una nota metodológica sobre la base de datos utilizada para analizar las características sociodemográficas de los migrantes de retorno y por último una reflexión sobre los retos y oportunidades que puede enfrentar México respecto a la población que retorna al país.

\section{Los estudios sobre migración de retorno en diversas latitudes y en México}

La migración de retorno no es un fenómeno nuevo, ni el estudio de la misma. Con diversos acercamientos teóricos y metodológicos se ha explicado los motivos del retorno principalmente a partir de la segunda mitad del siglo XX, desde estudios de corte histórico, económico, sociológico, demográfico y político. Muchos de los cuales fueron realizados en el continente europeo en la década de los sesenta y posteriormente se extienden hacia otras latitudes así dan cuenta el estudio de Fernández (2010 pag 28).

Algunos autores atribuyen el regreso como resultado de las dificultades por adaptarse al lugar de destino, o porque existen factores de atracción del país de origen, como King (1986), Durand (2004) o Fernández (2011). También existe el interés de saber si el migrante de retorno puede contribuir a su país, si pueden ser vehículo de desarrollo social (Cassarino 2004, Papail y Arroyo 2004, Rivera 2011, Nieto 2012). De tal manera que la migración de retorno se considera como una oportunidad de desarrollo para el país de origen del migrante, ya que puede desempeñar las capacidades, conocimientos y habilidades adquiridas en su trayectoria migratoria y laboral en el lugar de destino.

Sin embargo, para autores como Nieto (2012) al estudiar la migración en una provincia de Perú hacia Italia, encontró que no todos los migrantes acumulan capital humano en su experiencia migratoria y en ocasiones pierden conocimientos. A lo que llama pérdida relativa y absoluta del capital humano inicial (Nieto 2012, pag 64-65). Por su parte, Schramm (2011) considera central las redes sociales para el éxito del retorno de los migrantes, como lo mostró en su estudio sobre los ecuatorianos en España.

La magnitud de la migración de retorno, las características de la población y las condiciones internacionales y nacionales en la dinámica de los mercados laborales, dan pautas para acercandose al conocimiento de la inserción de los migrantes de retorno a su país de origen y sociedad que los acoge.

\subsection{La migración de retorno en México contem-} poráneo

Como se mencionó al inicio del artículo, la migración de mexicanos hacia Estados Unidos es añeja, compleja, dinámica y cambiante. Caracterizada desde el inicio como una migración masculina temporal y circular, el retorno 
de los migrantes fue desde el inicio una etapa más de la trayectoria migratoria de los mexicanos.

Gómez (2014) señala que entre 1908 y 1922 fue la primera etapa de retorno de migrantes de Estados Unidos a México, momentos determinados por los ciclos de la economía estadounidense principalmente en la Gran Depresión.

Con la firma del Programa Bracero (1942-1964), -acuerdo binacional entre México y Estados Unidos-, se institucionalizó la migración temporal y circular que respondía a la demanda de mano de obra de la Unión Americana. Al cancelarse unilateralmente en 1964 dicho Programa, hubo un volumen importante de migrantes que retornaron pero también se había constituido una cultura migratoria en varias regiones del país. De tal manera que, a pesar del retorno y cancelación del programa, ya se reconocía que había millones de mexicanos residiendo en el país vecino a finales de los años setenta (Gómez, 2014).

En la década de los años 80's, con la aprobación de la Ley de Inmigración Immigration Reform and Control Act (IRCA-1986), se legalizaron casi 3 millones de migrantes lo que posibilitó nuevas características al proceso migratorio mexicano, con nuevos migrantes, reunificación familiar, procedentes tanto de pueblos como ciudades, así que el perfil del migrante había cambiado, ahora se reconocía la existencia de la migración femenina y familiar con un patrón migratorio de mayor permanencia en Estados Unidos. Sin embargo, la migración de retorno no se detuvo y se empieza a registrar oficialmente en diferentes encuestas principalmente a partir de la década de los noventa como: la Encuesta Nacional de la Dinámica Demográfica (ENADID), la Encuesta Nacional de Ocupación y Empleo (ENOE), y el Censo Nacional de Población y Vivienda (CPV). La ENADID se aplica en viviendas y las unidades de observación son las personas; la ENOE se levanta en hogares y el CPV en las viviendas.

Ha llamado la atención en el ámbito académico y político el aumento de la migración de retorno en el nuevo milenio. La migración de retorno desde Estados Unidos a México tuvo su registro más alto en 2010; sin embargo, varios autores mencionan que los flujos de retorno no tuvieron alteraciones significativas después de la crisis económica (Aldo, Ordáz y Li Ng 2012:243); Canales (2012) plantea que "el principal impacto de la crisis (económica) no está en tanto en un posible retorno masivo de mexicanos, como en una virtual desaceleración de flujo de entrada de mexicanos hacia Estados Unidos" (Canales 2012, pag 119), aunque reconoce que existen otros factores que ayudan a entender el retorno en los últimos años como el ambiente antinmigrante, freno del crecimiento de la migración. Por su parte García y Zamora (2014) mencionan que hay un descenso en la migración de retorno que podría estar relacionada a la reducción de la emigración mexicana (2014, pág 217); López y Mojica señalan que "el retorno y las salidas son dos caras de la misma moneda: la migración. En algunas etapas histórica una ha prevalecido sobre la otra o incluso se han equilibrado" (López y Mojica 2013, pág 8).

No es que exista una contradicción sobre la estimación de la migración de retorno, la información responde a la muestra y población registrada. Lo que enfatiza Canales (2012) es que la proporción de la migración de retorno no ha variado considerablemente desde la década de los noventa. El punto de coincidencia de los autores referidos párrafo arriba, es que el volumen de la migración de retorno ha aumentado y nuestra hipótesis es que esta migración va continuar con diferentes comportamientos, de acuerdo a condiciones estructurales de México y Estados Unidos, pero también por los proyectos individuales y familiares de los migrantes. De ahí la relevancia de reconocer que se trata de una población heterogénea, de la cual debe estudiarse sus características sociodemográficas y económicas; debido a que puede tener impacto diferenciado en las localidades de asentamiento al regresar a México.

Existen recientes estudios con diversas metodologías y fuentes estadísticas que están aportando al conocimiento del migrante que retorna en la actualidad a México. Se podría decir que son tres dimensiones de análisis que se han privilegiado: en conocer las características del migrante retornado a nivel nacional y en algunos estados de la república mexicana (García y Zamora 2014; Albo, 
Ordáz y Li Ng 2012); la segunda, la vinculación que puede tener la migración de retorno con el desarrollo de las comunidades ya sea a través del capital financiero o capital humano (Papail y Arroyo 2004, Cobos, Giorguli y Alba 2010); y la tercera, el interés de analizar la inserción laboral del migrante en la sociedad de acogida (Anguiano, Cruz y Garbey 2013; Rivera 2011).

Albo, Ordáz y Li Ng (2012) mencionan que la población de retornados se dirige principalmente a localidades rurales, aunque existe un ligero aumento en localidades urbanas de acuerdo a las estadísticas de la ENOE. En cambio García y Zamora (2014) encontraron que el lugar a donde se dirigen los migrantes retornados son localidades urbanas, principalmente de Jalisco, Michoacán, Guanajuato, Sonora, Nuevo León, San Luis Potosí, Tamaulipas y Sinaloa; identificadas como regiones norte y tradicional (García y Zamora 2014:231), estos hallazgos responden al análisis de la Encuesta de la Migración Frontera Norte.

En otros estudios se destaca la importancia de los migrantes de retorno en las comunidades de origen. Ya sea por la inversión económica que propiciaba que el migrante retornado cambiara de ser trabajador asalariado, a trabajador por cuenta propia, o por el capital humano adquirido en Estados Unidos (Papail y Arroyo 2004; Fernández 2011; García y Zamora 2014).

De acuerdo a Albo, Ordáz y Li Ng (2012) los migrantes que retornan a localidades urbanas, $75 \%$ tarda 3 meses en emplearse y en menor proporción 64\%. Si bien, se podría considerar que el tiempo para emplearse es relativamente corto, estudios como los de Anguiano, Cruz y Garbey (2013) encontraron que no existe una reinserción exitosa en el mercado laboral de los migrantes que regresaron a Veracruz. Rivera (2011) presenta resultados similares en una localidad del Estado de México, en la mayoría de los retornados no significó una movilidad ascendente. Por su parte García y Zamora (2014) encontraron una correspondencia en las ocupaciones realizadas en Estados Unidos y en México. El estudio de Cobos, Giorguli y Alba (2010) demuestran que en algunos casos la experiencia migratoria de los mexicanos no necesariamente acumula activos, de tal manera que "no todas las experiencias migratorias laborales en el extranjero contienen elementos de acumulación de activos que se traduzcan en una movilidad ascendente" (Cobos, Giorguli y Alba 2010, pág 270).

Los recientes estudios sobre migración de retorno presentan la complejidad del análisis dependiendo del nivel de escala que se considera, a nivel nacional, estado o comunidad. De ahí la necesidad de conocer que características predominan a nivel nacional, regional y entidades federativas que registraron mayor volumen de migrantes de retorno.

\section{Metodología}

El Censo de Población y Vivienda es una de las fuentes de información más confiables en términos migratorios por su cobertura nacional, por entidad, municipio y tamaño de localidad. Lo que permite un acercamiento a la población objeto de estudio, que en nuestro caso son los migrantes de retorno y algunas de sus principales características demográficas y económicas. Para el censo de 2010 el instrumento de captación fueron dos cuestionarios: uno básico para obtener información sobre la vivienda, hogares y las personas, y otro dirigido a precisar algunos datos sobre las viviendas y las personas, que incluyó preguntas sobre la migración internacional en los últimos cinco años.

El cuestionario base ofrece más información de los migrantes de retorno, los identifica al preguntar el lugar de residencia hace 5 años y lugar de nacimiento. Se denomina como migrante de retorno a las personas que señalaron durante la entrevista del CPV de 2010, haber nacido en México y radicaban en Estados Unidos hace cinco años, esta es la población objeto de estudio. Los casos ponderados fueron 825,609 a nivel nacional y la información se obtuvo hasta junio del año de aplicación. Las variables analizadas son: edad, sexo, estado civil, escolaridad, así como algunas de sus características económicas como ocupación e ingreso, inclusive interesa identificar el acceso al servicio de salud de la población de 
retorno porque se parte del supuesto que este indicador es importante para el proceso de integración de la población que retorna a sus comunidades, además de la jefatura y condición de parentesco, la ocupación y la educación.

La información se presenta en diferentes escalas, a nivel nacional, regional y estatal. La desagregación en estas escalas permite conocer las especificidades de la migración de retorno e identificar los posibles retos que enfrentará la población que regresa a la sociedad de asentamiento y cuáles pueden ser las agendas pendientes de políticas públicas para atender a las necesidades de los y las migrantes de retorno.

¿Por qué considerar la escala regional? Como se ha mencionado, en México se considera un país de emigrantes porque se registra que las personas proceden de casi todas las entidades federativas del país; sin embargo, esta movilización responde a procesos y contextos diferentes, de ahí que varios autores como Durand y Massey (2004) así como el Consejo Nacional de Población (CONAPO) han establecido una regionalización sobre los lugares de origen de los migrantes mexicanos que van a Estados Unidos, de tal manera que se articulan criterios geográficos y población migratoria. CONAPO (2012) subdivide al territorio mexicano en cuatro regiones: Tradicional, Norte, Centro y Sur-sureste (Mapa 1).

\section{Mapa 1. Regiones migratorias de México}

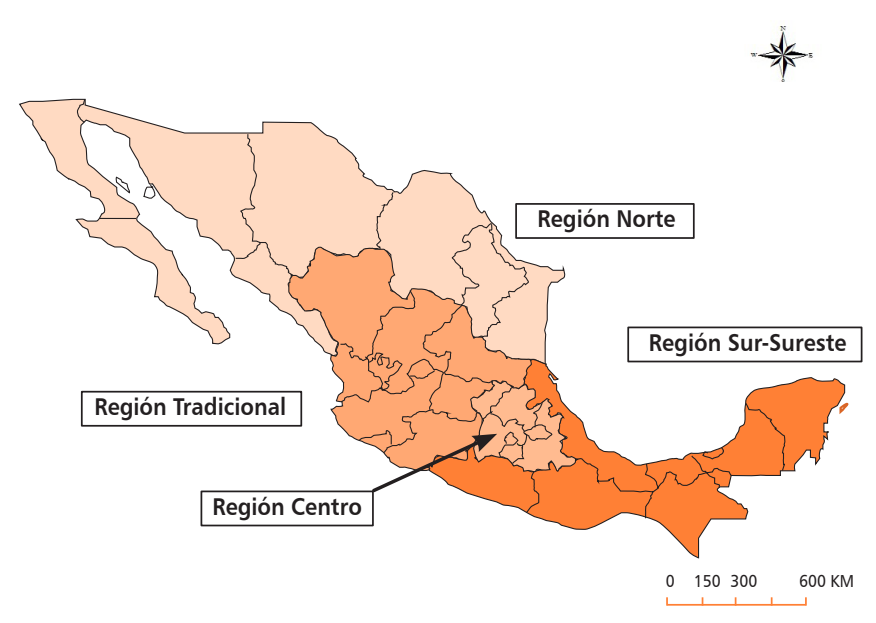

Elaboración propia con base en la clasificación hecha por CONAPO.
- La Región Tradicional destaca por ser el origen principal de la corriente migratoria mexicana a Estados Unidos. Son nueve entidades del centro-occidente del país: Aguascalientes, Colima, Durango, Guanajuato, Jalisco, Michoacán, Nayarit, San Luis Potosí y Zacatecas. Son los estados de migración más añeja, donde se estableció lo que algunos autores llaman cultura migratoria y redes consolidadas.

- La Región Centro se distingue por un fuerte dinamismo migratorio, sobre todo a partir de la década de 1980. Se ubica el estado de Morelos, Querétaro, Tlaxcala, Puebla, Hidalgo, Distrito Federal y el Estado de México. Se destaca el aumento del flujo migratorio para el caso de Morelos y del estado de Hidalgo, donde la migración involucra a un número cada vez mayor de municipios y comunidades.

- La Región Sur-sureste incluye a ocho entidades federativas: Campeche, Chiapas, Guerrero, Oaxaca, Quintana Roo, Tabasco, Veracruz y Yucatán. Esta región se distingue por su reciente incorporación a la migración hacia Estados Unidos, la cual inicia fundamentalmente a partir de la década de 1990, con excepción de Guerrero y Oaxaca, cuyos orígenes se remontan a los años cuarenta en el contexto del Programa Bracero.

- La Región Norte ha tenido una participación significativa en la migración a Estados Unidos, seis de las ocho entidades que la componen tienen frontera con Estados Unidos: Baja California, Coahuila, Chihuahua, Nuevo León, Sonora y Tamaulipas. Incluye, además, dos estados que mantienen vínculos geográficos y migratorios con los estados fronterizos: Baja California Sur y Sinaloa. Un rasgo característico de esta región es la migración transfronteriza (commuters); es decir, personas que viven y/o trabajan en ambos lados de la frontera.Es además receptora de grandes flujos migratorios provenientes del interior del país y de aquellos migrantes mexicanos que son devueltos por las autoridades migratorias estadounidenses.

En este artículo interesa presentar el volumen y características de la migración de retorno por regiones, destacando estados en los que se distinguen algunas varia- 
bles, para ver su relación con las regiones y si son propias de la entidad federativa. Cabe señalar que al desagregar por tamaño de localidad se considera población urbana a la población que habita en localidades de 15,000 o más habitantes como ha sido establecido por el Instituto de Geografía Estadística e Informática (INEGI).

\section{Regiones y entidades principales de los migrantes internacionales retornados}

La región que mayor proporción de migrantes de retorno registran es la región tradicional. Cabe destacar que no sólo se caracteriza por ser la emigración más añeja, sino también porque los estados que la comprenden son los que mayor volumen de migrantes hacia Estados Unidos registran, principalmente Jalisco, Michoacán, Guanajuato y Zacatecas. Le sigue casi con el mismo porcentaje, la Región Norte y Centro. Eso podría estar relacionado con el planteamiento de Canales (2012) de que existe una correspondencia del volumen de emigración con la población de retorno (Cuadro1).
Cuadro 1. Migrantes de retorno por sexo y regiones migratorias

\begin{tabular}{|l|r|r|r|r|r|}
\multirow{2}{*}{ Sexo } & \multicolumn{4}{|c|}{ Regiones Migratorias } & \multirow{2}{*}{ Total } \\
\cline { 2 - 5 } & Tradicional & Centro & Sur-Sureste & Norte & \multicolumn{1}{c|}{ Tot } \\
\hline Hombre & $28,2 \%$ & $16,4 \%$ & $13,2 \%$ & $14,2 \%$ & $72,0 \%$ \\
\hline Mujer & $10,0 \%$ & $5,5 \%$ & $4,6 \%$ & $7,9 \%$ & $28,0 \%$ \\
\hline Total & $38,2 \%$ & $21,9 \%$ & $17,8 \%$ & $22,0 \%$ & $100,0 \%$ \\
\hline
\end{tabular}

Fuente: Elaboración propia con base en el microdato de la base PERS del censo de población y Vivienda 2010, INEGI.

Las principales entidades federativas con migrantes retornados fueron: Jalisco (8.7\%), Michoacán (8.4\%), Guanajuato $(7.9 \%)$, que corresponden a la región tradicional; Estado de México (6.3\%) a la región centro; y Veracruz (5.9\%) de la región del sur-sureste. Les siguen Baja California (4.9\%), Guerrero, Oaxaca, Puebla y Chihuahua (4.3\%) respectivamente. Cabe destacar que cuando se observa la proporción de hombres y mujeres los porcentajes más elevados son para el estado de Jalisco, Michoacán, Guanajuato de la región tradicional, Chihuahua, Baja California, de la región norte, Estado de México región centro y Veracruz región sur-sureste (Gráfico 1).

\section{Gráfico 1. Migrantes de retorno por entidad y sexo en 2010}

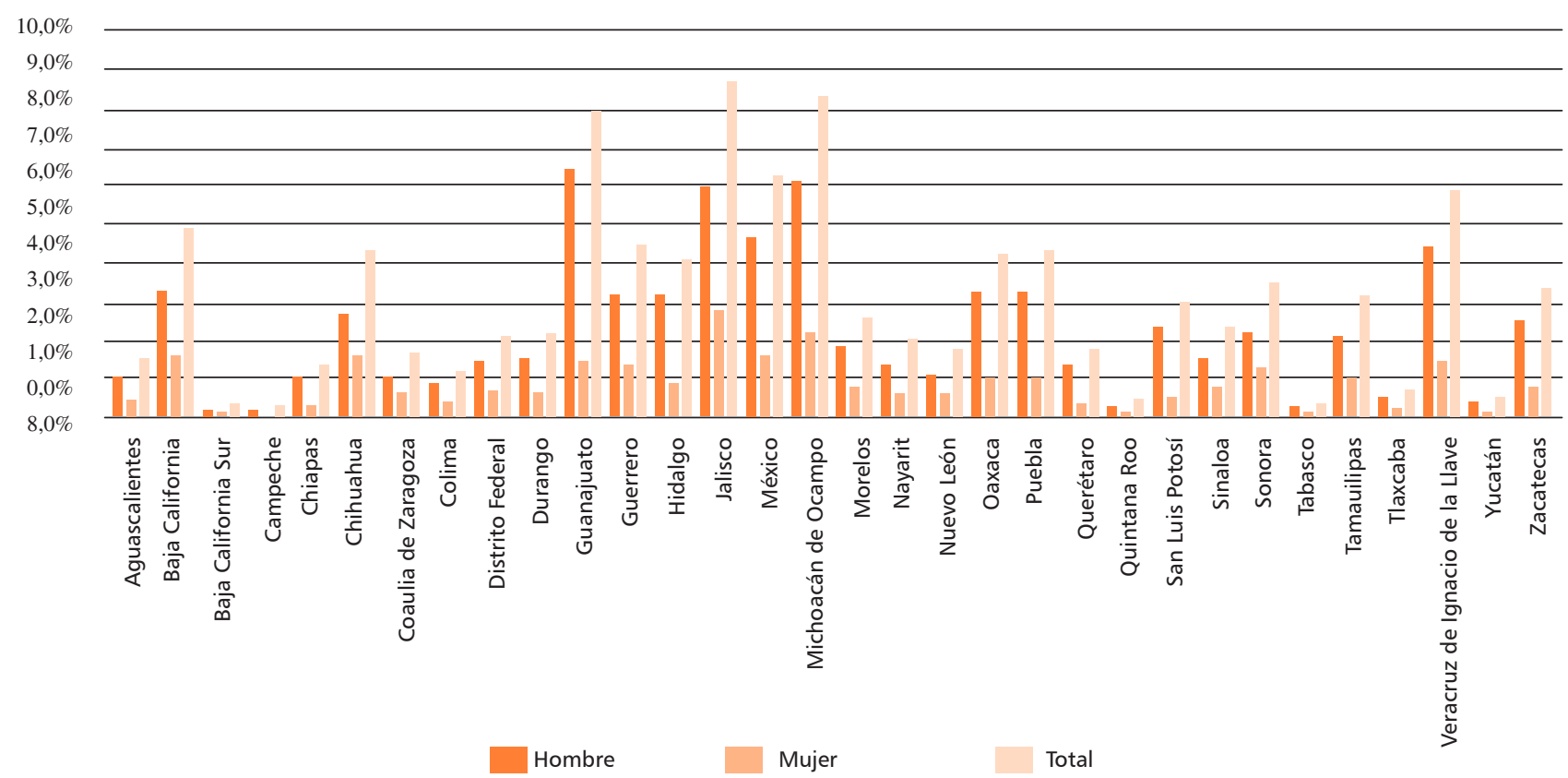

Fuente: Elaboración propia con base en el micro-dato de la base PERS del Censo de Población y Vivienda 2010, INEGI 
García y Zamora (2014) señalan que existe una subestimación en el registro de las mujeres que retornan, pero también se ha demostrado que las mujeres prefieren permanecer en Estados Unidos a diferencia del hombre (Woo, 2007). De tal manera que existen diferencias de género tanto para emigrar como para retornar, más adelante se retoma el análisis de la diferencia por género a otras escalas.

Las encuestas que captan los motivos de retorno son la ENOE y la EMIF, de acuerdo al análisis de estas bases de datos se han identificado diversos motivos para regresar tales como: trabajo, casamiento, unión, separación, reunificación familiar problemas de salud y deportación (Albo, Ordáz y Li Ng 2012; García y Zamora 2014).

\subsection{Tamaño de localidad y migración de retorno}

Respecto al tamaño de localidad, de acuerdo al CPV 2010, se encontró que a nivel nacional, la mayoría de los migrantes de retorno llegan a localidades menores de 15,000 habitantes con 53.4\%; le sigue en importancia localidades mayores a 100 mil habitantes con $31.2 \%$; y posteriormente las localidades de 15,000 a 99,999 con casi $16 \%$.

Conocer a dónde llegan los migrantes retornados es muy importante, se ha encontrado que el contexto de estas localidades puede ayudar o inhibir las posibilidades de incorporarse al mercado laboral así como al acceso a la educación y salud, sin dejar de mencionar el impacto cultural tanto para el que regresa como para la comunidad que los recibe.

Respecto a las entidades federativas se encontró que existe un predominio de retorno a localidades rurales en el caso de Veracruz (71.5\%), Michoacán (66.4\%) y Guanajuato (64.6\%), es decir, a localidades menores a 15 mil habitantes (Cuadro 2).

En Jalisco es diferente, prácticamente la mitad de dicha población migrante regresó a localidades menores de 15,000 habitantes $(51,2 \%)$ y la otra mitad a localidades de más urbanización, con más 15 mil habitantes (48.8\%). Situación similar para el Estado de México pero en localidades de mayor población, 48\% regresó a localidades de 100,000 habitantes.

Cabe mencionar que tanto en Jalisco como en el Estado de México se encuentran dos ciudades importantes: Guadalajara en Jalisco, que destaca por sus actividades comerciales, industriales, así como culturales; y Toluca como una de las principales ciudades industriales del Estado de México y del centro del país. Ambas ciudades siempre han tenido una importante atracción de población.

Cuadro 2. Distribución migrantes de retorno por tamaño de localidad y entidad en 2010

\begin{tabular}{|c|c|c|c|c|c|c|c|c|c|c|c|c|}
\hline \multirow[b]{3}{*}{ Tamaño de localidad } & \multicolumn{6}{|c|}{ Dentro de Nombre de la entidad federativa } & \multicolumn{6}{|c|}{ Dentro de Tamaño de localidad } \\
\hline & \multicolumn{5}{|c|}{ Nombre de la entidad federativa } & \multirow[b]{2}{*}{ Total } & \multicolumn{5}{|c|}{ Nombre de la entidad federativa } & \multirow[b]{2}{*}{ Total } \\
\hline & 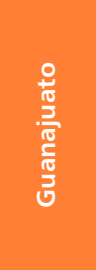 & $\begin{array}{l}\text { 8 } \\
\frac{\text { m }}{\mathrm{N}}\end{array}$ & $\frac{8}{\frac{3}{x}}$ & 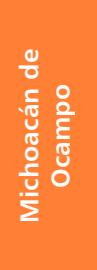 & 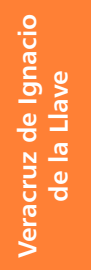 & & 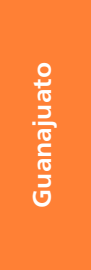 & 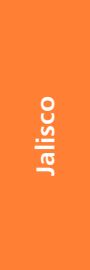 & $\begin{array}{l}\stackrel{8}{\mathrm{~J}} \\
\frac{\mathbb{x}}{\Sigma}\end{array}$ & 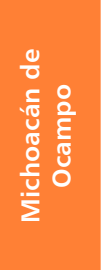 & 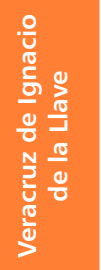 & \\
\hline Menos de 2500 habitantes & $51,0 \%$ & $27,4 \%$ & $22,6 \%$ & $41,0 \%$ & $51,1 \%$ & $38,4 \%$ & $28,3 \%$ & $16,7 \%$ & $9,9 \%$ & $24,0 \%$ & $21,1 \%$ & $100 \%$ \\
\hline 2500 a 14999 habitantes & $13,6 \%$ & $23,8 \%$ & $16,5 \%$ & $25,4 \%$ & $20,4 \%$ & $20,2 \%$ & $14,3 \%$ & $27,5 \%$ & $13,8 \%$ & $28,3 \%$ & $16,0 \%$ & $100 \%$ \\
\hline 15000 a 99999 habitantes & $18,6 \%$ & $24,3 \%$ & $12,5 \%$ & $19,8 \%$ & $14,0 \%$ & $18,5 \%$ & $21,5 \%$ & $30,9 \%$ & $11,4 \%$ & $24,1 \%$ & $12,1 \%$ & $100 \%$ \\
\hline 100000 y más habitantes & $16,8 \%$ & $24,5 \%$ & $48,5 \%$ & $13,8 \%$ & $14,4 \%$ & $22,9 \%$ & $15,7 \%$ & $25,0 \%$ & $35,7 \%$ & $13,6 \%$ & $10,0 \%$ & $100 \%$ \\
\hline Total & $100 \%$ & $100 \%$ & $100 \%$ & $100 \%$ & $100 \%$ & $100 \%$ & $21,4 \%$ & $23,4 \%$ & $16,9 \%$ & $22,5 \%$ & $15,9 \%$ & $100 \%$ \\
\hline
\end{tabular}

Fuente: Elaboración propia con base en el microdato de la base PERS del Censo de Población y Vivienda 2010, INEGI 
4.2 La categoría sexo/género de la población de retorno

El sexo es más que una variable, si se analiza con la categoría de género, es posible darse cuenta que poco se hace referencia a la diferencia que existe en la emigración y retorno de los hombres y las mujeres. De acuerdo al CPV del 2010, del total de retornados, $72 \%$ eran hombres y $28 \%$ eran mujeres. Se podría decir que es una migración de retorno selectiva por sexo, ya en otros estudios con diversas metodologías se ha encontrado que las mujeres tienen mayor propensión a permanecer por tiempo más prolongado o definitivo en Estados Unidos, ya que para ellas, las oportunidades laborales y de bienestar son mayores que en México (Woo, 2007).

Esta condición de género les lleva a vivir la experiencia de migrar y de retornar de manera diferenciada a donde llegan. De acuerdo al CPV 2010, los hombres regresaron en mayor proporción a las localidades rurales (27.1\%) y las mujeres lo hicieron más a las ciudades (Gráfica 2). El acceso a oportunidades para el bienestar social no sólo es diferente entre los hombres y las mujeres sino también en las oportunidades que pueden brindarles la localidad en donde llegan, para insertarse laboralmente así como otros factores y procesos de más complejidad a nivel estatal y regional, factores que pueden converger como la migración interna, pobreza, acceso a seguridad social y educación, articulación de redes sociales, entre otros elementos.
Gráfico 2. Migrantes de retorno por número de habitantes de localidad y sexo en 2010

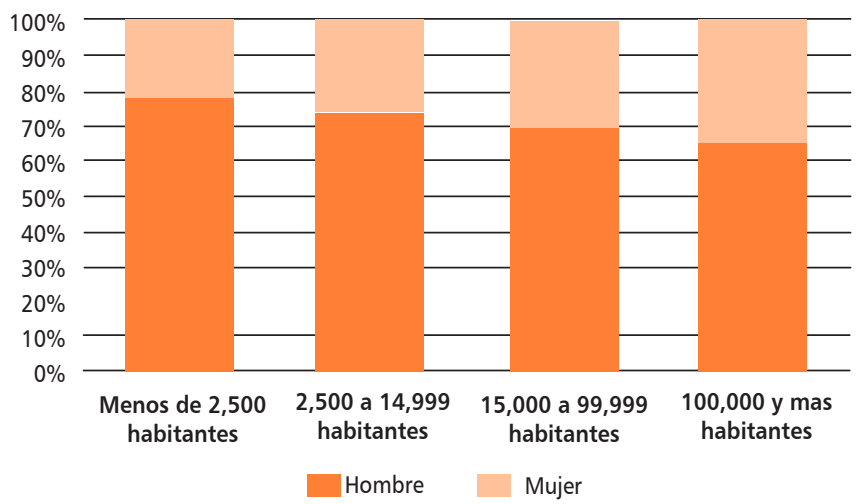

Fuente: Elaboración propia con base en el micro-dato de la base PERS del Censo de Población y Vivienda 2010, INEGI

\subsection{Edad de los migrantes retornados}

De acuerdo al CPV 2010, se encontró a nivel nacional, regional y los estados referidos en este artículo, que existe coincidencia con los estudios que citan una participación significativa de migrantes retornados en edad productiva. Con $69.4 \%$ en los rangos de edad de 21 a 40, y 20.7\% de 41 a 60 años. Sin embargo, se considera importante destacar la población menor de 20 años con un porcentaje acumulado que oscila entre $8 \%$ en Guanajuato, hasta 11.7\% Jalisco (Cuadro 3). Ya que se ha marcado la relevancia de la población en edad productiva, pero no se debe soslayar que el regreso de niños, jóvenes y adultos mayores presentarán diversas

Cuadro 3. Grupos de edad de la población migrante retornada en 2010

\begin{tabular}{|c|c|c|c|c|c|c|}
\hline & \multicolumn{5}{|c|}{ Nombre de la entidad federativa } & \multirow{2}{*}{ Total } \\
\hline & Guanajuato & Jalisco & Estado de México & Michoacán & Veracruz & \\
\hline De 0 a 10 años & $1,8 \%$ & $3,5 \%$ & $2,6 \%$ & $2,9 \%$ & $2,2 \%$ & $2,6 \%$ \\
\hline De 11 a 20 años & $6,2 \%$ & $8,2 \%$ & $8,2 \%$ & $6,7 \%$ & $6,1 \%$ & $7,1 \%$ \\
\hline De 21 a 30 años & $37,3 \%$ & $32,4 \%$ & $32,5 \%$ & $36,0 \%$ & $33,0 \%$ & $34,4 \%$ \\
\hline De 31 a 40 años & $31,3 \%$ & $32,3 \%$ & $35,5 \%$ & $30,2 \%$ & $34,0 \%$ & $32,4 \%$ \\
\hline De 41 a 50 años & $13,3 \%$ & $12,7 \%$ & $13,0 \%$ & $12,6 \%$ & $17,2 \%$ & $13,6 \%$ \\
\hline De 51 a 60 años & $6,7 \%$ & $5,4 \%$ & $5,9 \%$ & $7,1 \%$ & $6,8 \%$ & $6,4 \%$ \\
\hline \multirow[t]{2}{*}{ Más de 60 años } & $3,4 \%$ & $5,5 \%$ & $2,3 \%$ & $4,5 \%$ &, $8 \%$ & $3,5 \%$ \\
\hline & $100,0 \%$ & $100,0 \%$ & $100,0 \%$ & $100,0 \%$ & $100,0 \%$ & $100,0 \%$ \\
\hline
\end{tabular}

Fuente: Elaboración propia con base en el micro-dato de la base PERS del Censo de Población y Vivienda 2010, INEGI. 
necesidades para reinsertarse a la sociedad, tanto al sistema educativo como al sistema de salud, aspectos que se abordarán más adelante.

\subsection{Escolaridad de los migrantes retornados}

La literatura se ha concentrado más sobre la selectividad educativa tanto del que emigra como del que regresa, tratando de vincularlo con el desarrollo de las localidades de origen y/o de retorno. A nivel nacional, respecto a la población que regresó de Estados Unidos, los mayores porcentajes se ubican en la educación básica, siendo el nivel primaria y secundaria los porcentajes más altos con $36.3 \%$ y $35.4 \%$ respectivamente. Con bachillerato $18 \%$ y licenciatura 5.6\% (Cuadro 4).

\section{Cuadro 4. Último Grado Aprobado en 2010 por sexo}

\begin{tabular}{|l|r|r|r|}
\multirow{2}{*}{$\begin{array}{c}\text { Ultimo grado aprobado } \\
\text { pro sexo, 2010 }\end{array}$} & \multicolumn{3}{|c|}{ Sexo } \\
\cline { 2 - 4 } Ninguno & Hombre & \multicolumn{1}{|c|}{ Mujer } & \multicolumn{1}{c|}{ Total } \\
\hline Preescolar & $2,1 \%$ &, $6 \%$ & $2,7 \%$ \\
\hline Primaria & $27,1 \%$ & $9,2 \%$ & $36,3 \%$ \\
\hline Secundaria & $26,1 \%$ & $9,3 \%$ & $35,4 \%$ \\
\hline Preparatoria o bachillerato & $12,3 \%$ & $5,7 \%$ & $18,0 \%$ \\
\hline Licenciatura o profesional & $3,3 \%$ & $2,3 \%$ & $5,6 \%$ \\
\hline Posgrado &, $4 \%$ &, $2 \%$ &, $6 \%$ \\
\hline No especificado &, $2 \%$ &, $1 \%$ &, $3 \%$ \\
\hline & $72,1 \%$ & $27,9 \%$ & $100,0 \%$ \\
\hline
\end{tabular}

Fuente: Elaboración propia con base en el micro-dato de la base PERS del Censo de Población y Vivienda 2010, INEGI.
Si bien existen muchas similitudes a nivel nacional y estatal, cuando se analiza por entidad federativa se encuentra que Jalisco (3.4\%), Estado de México (3.2\%) y Veracruz (3\%) tienen mayor proporción de población con bachillerato. Los porcentajes más elevados en orden de importancia se encuentran en Guanajuato, Michoacán y Jalisco (Cuadro 5). No existe correspondencia en estos últimos niveles escolares en las variables a nivel nacional y estado.

\subsection{Jefatura y parentesco de los migrantes que retornaron}

Otra de las características sociales que se analizó en las entidades con mayores porcentajes de migración de retorno, fue la jefatura y la relación parental que tenían otros miembros que retornaron respecto del jefe o jefa del hogar del migrante. De las cinco entidades, Veracruz y Guanajuato cuentan con un porcentaje más alto de jefes de hogar $(88.2 \%$ y $84.1 \%$ respectivamente). Aunque predominaron los hombres, es más alta la proporción de mujeres jefas de hogar de estas entidades federativas, $23.3 \%$ y $21.3 \%$ respectivamente (Cuadro 6).

En Veracruz (36.7\%) y Guanajuato (40.1\%) llegaron menos mujeres en condición de esposas; mientras que en Michoacán (46.6\%) y Jalisco (45.2\%) sucedió lo contrario, había más mujeres en condición de esposas.

\section{Cuadro 5. Niveles de educación de los migrantes de retorno en 2010 por entidades}

\begin{tabular}{|c|c|c|c|c|c|c|}
\hline \multirow[b]{2}{*}{ Nivel } & \multicolumn{5}{|c|}{ Nombre de la entidad federativa } & \multirow[b]{2}{*}{ Total } \\
\hline & Guanajuato & Jalisco & México & $\begin{array}{l}\text { Michoacán de } \\
\text { Ocampo }\end{array}$ & $\begin{array}{l}\text { Veracruz de } \\
\text { Ignacio de la } \\
\text { Llave }\end{array}$ & \\
\hline Ninguno & $1,1 \%$ &, $5 \%$ &, $3 \%$ & $1,1 \%$ &, $5 \%$ & $3,5 \%$ \\
\hline Preescolar & $3 \%$ &, $2 \%$ &, $2 \%$ & $3 \%$ &, $2 \%$ & $1,2 \%$ \\
\hline Primaria & $10,0 \%$ & $9,7 \%$ & $4,5 \%$ & $10,6 \%$ & $6,1 \%$ & $40,7 \%$ \\
\hline Secundaria & $6,8 \%$ & $7,6 \%$ & $6,8 \%$ & $6,9 \%$ & $4,8 \%$ & $32,9 \%$ \\
\hline Preparatoria o bachillerato & $2,4 \%$ & $3,4 \%$ & $3,2 \%$ & $2,7 \%$ & $3,0 \%$ & $14,7 \%$ \\
\hline $\begin{array}{l}\text { Estudios técnicos o comerciales con primaria, } \\
\text { secundario o bachillerato terminado }\end{array}$ &, $2 \%$ &, $7 \%$ &, $6 \%$ &, $3 \%$ &, $2 \%$ & $1,1 \%$ \\
\hline Normal, Licenciatura o profesional &, $5 \%$ & $1,0 \%$ &, $9 \%$ &, $5 \%$ & $1,0 \%$ & $4,1 \%$ \\
\hline Posgrado &, $0 \%$ &, $2 \%$ &, $2 \%$ &, $0 \%$ &, $0 \%$ & $.4 \%$ \\
\hline \multirow[t]{2}{*}{ No especificado } &, $0 \%$ & , 1\% &, $0 \%$ &, $0 \%$ &, $0 \%$ &, $2 \%$ \\
\hline & $21,4 \%$ & $23,4 \%$ & $16,9 \%$ & $22,5 \%$ & $15,9 \%$ & $100,0 \%$ \\
\hline
\end{tabular}

Fuente: Elaboración propia con base en el micro-dato de la base PERS del Censo de Población y Vivienda 2010, INEGI 
Cuadro 6. Jefatura y parentescos de los migrantes retornados en 2010

\begin{tabular}{|c|c|c|c|c|c|c|c|c|c|c|}
\hline & \multicolumn{2}{|c|}{ Guanajuato } & \multicolumn{2}{|c|}{ Jalisco } & \multicolumn{2}{|c|}{ México } & \multicolumn{2}{|c|}{$\begin{array}{l}\text { Michoacán } \\
\text { de Ocampo }\end{array}$} & \multicolumn{2}{|c|}{$\begin{array}{c}\text { Veracruz de Ignacio } \\
\text { de la Llave }\end{array}$} \\
\hline & \multicolumn{2}{|c|}{ Sexo } & \multicolumn{2}{|c|}{ Sexo } & \multicolumn{2}{|c|}{ Sexo } & \multicolumn{2}{|c|}{ Sexo } & \multicolumn{2}{|c|}{ Sexo } \\
\hline & Hombre & Mujer & Hombre & Mujer & Hombre & Mujer & Hombre & Mujer & Hombre & Mujer \\
\hline Jefa(e) & $62,8 \%$ & $21,3 \%$ & $57,5 \%$ & $17,2 \%$ & $59,8 \%$ & $14,5 \%$ & $61,8 \%$ & $17,5 \%$ & $64,9 \%$ & $23,3 \%$ \\
\hline $\begin{array}{l}\text { Esposa(o) o } \\
\text { compañera(o) }\end{array}$ & $2,3 \%$ & $40,1 \%$ & $2,0 \%$ & $45,2 \%$ & $2,2 \%$ & $41,6 \%$ & $1,8 \%$ & $46,6 \%$ & $1,8 \%$ & $36,7 \%$ \\
\hline $\mathrm{Hija}(\mathrm{o})$ & $26,4 \%$ & $26,9 \%$ & $28,3 \%$ & $24,7 \%$ & $25,7 \%$ & $30,1 \%$ & $28,1 \%$ & $23,4 \%$ & $24,6 \%$ & $24,6 \%$ \\
\hline Nieta(o) & $1,4 \%$ & $1,9 \%$ & $2,5 \%$ & $2,3 \%$ & $2,0 \%$ & $3,5 \%$ & $1,7 \%$ & $2,8 \%$ & $1,8 \%$ & $3,5 \%$ \\
\hline Nuera o yerno & $2,8 \%$ & $5,1 \%$ & $3,0 \%$ & $3,5 \%$ & $4,0 \%$ & $4,3 \%$ & $2,4 \%$ & $5,5 \%$ & $3,1 \%$ & $6,0 \%$ \\
\hline Madre o padre & $0,0 \%$ & $0,8 \%$ & $0,1 \%$ & $1,1 \%$ & $0,1 \%$ & $0,8 \%$ & $0,1 \%$ & $0,7 \%$ & $0,1 \%$ & $0,4 \%$ \\
\hline Suegra(o) & $0,1 \%$ & $0,1 \%$ & $0,1 \%$ & $0,2 \%$ & $0,1 \%$ & $1,0 \%$ & $0,1 \%$ & $0,3 \%$ & $0,0 \%$ & $0,3 \%$ \\
\hline Otro parentesco & $3,5 \%$ & $3,7 \%$ & $5,6 \%$ & $4,8 \%$ & $5,2 \%$ & $2,8 \%$ & $3,1 \%$ & $2,8 \%$ & $2,6 \%$ & $4,1 \%$ \\
\hline Sin parentesco & $0,1 \%$ & $0,1 \%$ & $0,7 \%$ & $0,8 \%$ & $0,4 \%$ & $0,0 \%$ & $0,3 \%$ & $0,1 \%$ & $0,7 \%$ & $1,1 \%$ \\
\hline No especificado & $0,5 \%$ & - & $0,3 \%$ & $0,2 \%$ & $0,4 \%$ & $1,3 \%$ & $0,5 \%$ & $0,3 \%$ & $0,3 \%$ & $0,0 \%$ \\
\hline Totales & $100 \%$ & $100 \%$ & $100 \%$ & $100 \%$ & $100 \%$ & $100 \%$ & $100 \%$ & $100 \%$ & $100 \%$ & $100 \%$ \\
\hline
\end{tabular}

Fuente: Elaboración propia con base en el micro-dato de la base PERS del Censo de Población y Vivienda 2010, INEGI

La condición de parentesco (nueras o yernos, así como de nietos) es una categoría que permite reflexionar sobre el migrante retornado y el perfil familiar de quienes regresaron. En Veracruz y Estado de México registraron los porcentajes más altos respecto de las otras entidades, 9.1\% y $5.3 \%$ respectivamente (Cuadro 6).

Algo similar sucede con aquellos que tienen un parentesco no lineal con el jefe o jefa de hogar. En Jalisco (10.4\%) y Estado de México (8\%) es más elevado el porcentaje de otros parentescos o no parentesco que los vincula a los jefes de hogar (Cuadro 6). La presencia de yernos, nueras, nietos y nietas, así como otros parentescos y no parentescos, dan indicios sobre la composición de las familias de quienes retornaron, y podrían estar reflejando una variabilidad del tipo de familias (extensas y compuestas).

\subsection{Estado civil de los migrantes que retornaron}

Los estudios que se han realizado en la actualidad sobre migración de retorno destacan la participación de migrantes de retorno casados, condición que prevalece cuando se observa a nivel estatal; sin embargo, es interesante observar las diferencias de esta categoría por condición de unión. Destaca el caso de Veracruz en unión libre y el caso de Guanajuato, Michoacán y Jalisco pre- domina casado por lo civil y religiosamente (Ver Cuadro 7), eso podría responder a que estos últimos estados se han caracterizado por un predominio de cultura religiosa. También es interesante observar que el mayor porcentaje de migrantes de retorno solteros o solteras es para Jalisco con el 26.4\% (Cuadro 7).

\subsection{Atención médica de migrantes retornados}

Sobre la atención médica de los migrantes retornados de las cinco entidades federativas, la mayoría se atiende en los Sistemas de Salud Estatales. Cabe señalar el elevado porcentaje que se atienden en clínicas u hospitales privados los porcentajes mayores están en Michoacán (43\%), Veracruz (39.2\%) y Estado de México (39\%) eso debe tomarse con cuidado, porque en México predominan servicios de atención médica a bajo costo en farmacias, ${ }^{1}$ se puede inferir que la población recurre a estos espacios de atención medica ante la falta de cobertura del sistema de salud en México. También es importante destacar el elevado porcentaje en algunas entidades federativas que declara que no se atiende, como son los casos de Guanajuato, Jalisco y Michoacán (Cuadro 8).

1. En México se prohibió la venta de antibióticos sin receta médica, las farmacias empezaron a poner consultorios que atendían médicos a bajo costo- (desde 30 hasta 50 pesos) 
Cuadro 7. Distribución del estado civil por entidad para 2010

\begin{tabular}{|c|c|c|c|c|c|c|}
\hline \multirow[b]{2}{*}{ Estado Civil } & \multicolumn{5}{|c|}{ Nombre de la entidad federativa } & \multirow[b]{2}{*}{ Total } \\
\hline & Guanajuato & Jalisco & $\begin{array}{l}\text { Estado de } \\
\text { México }\end{array}$ & Michoacán & Veracruz & \\
\hline Unión libre & $14,7 \%$ & $16,3 \%$ & $25,1 \%$ & $17,2 \%$ & $32,6 \%$ & $20,2 \%$ \\
\hline Separada(o) & $3,1 \%$ & $4,3 \%$ & $7,3 \%$ & $4,1 \%$ & $6,0 \%$ & $4,8 \%$ \\
\hline Divorciada(o) & $2,1 \%$ & $2,4 \%$ & $1,4 \%$ & $1,4 \%$ & $2,4 \%$ & $2,0 \%$ \\
\hline Viuda(o) & $1,3 \%$ & $1,9 \%$ & $1,3 \%$ & $1,3 \%$ & ,7\% & $1,4 \%$ \\
\hline Casada(o) sólo por el civil & $12,3 \%$ & $13,7 \%$ & $13,8 \%$ & $16,6 \%$ & $17,3 \%$ & $14,7 \%$ \\
\hline Casada(o) sólo religiosamente & $1,3 \%$ & $1,4 \%$ & $2,4 \%$ & $2,2 \%$ & $4,1 \%$ & $2,1 \%$ \\
\hline Casada(o) civil y religiosamente & $44,2 \%$ & $33,5 \%$ & $24,9 \%$ & $37,0 \%$ & $16,0 \%$ & $32,4 \%$ \\
\hline Soltera(o) & $20,9 \%$ & $26,4 \%$ & $23,6 \%$ & $20,0 \%$ & $20,6 \%$ & $22,4 \%$ \\
\hline No especificado &, $1 \%$ &, $1 \%$ &, $1 \%$ &, $2 \%$ &, $1 \%$ &, $1 \%$ \\
\hline Totales & $100,0 \%$ & $100,0 \%$ & $100,0 \%$ & $100,0 \%$ & $100,0 \%$ & $100,0 \%$ \\
\hline
\end{tabular}

Fuente: Elaboración propia con base en el micro-dato de la base PERS del Censo de Población y Vivienda 2010, INEGI

Cuadro 8. Atención médica de los migrantes de retorno para 2010

\begin{tabular}{|c|c|c|c|c|c|c|}
\hline \multirow[b]{3}{*}{$\begin{array}{l}\text { Lugar o ins- } \\
\text { titución de } \\
\text { atención }\end{array}$} & \multicolumn{6}{|c|}{ Dónde se atiende según lugar o institución } \\
\hline & \multicolumn{5}{|c|}{ Nombre de la entidad federativa } & \multirow[b]{2}{*}{ Total } \\
\hline & 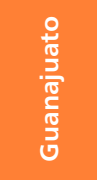 & 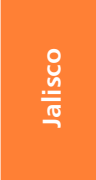 & 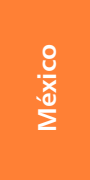 & 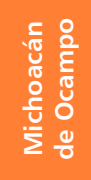 & 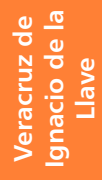 & \\
\hline $\begin{array}{l}\text { Seguro Social } \\
\text { (IMSS) }\end{array}$ & $15,9 \%$ & $31,0 \%$ & $18,8 \%$ & $19,1 \%$ & $15,3 \%$ & $100 \%$ \\
\hline ISSSTE & $13,1 \%$ & $13,3 \%$ & $15,1 \%$ & $23,3 \%$ & $35,2 \%$ & $100 \%$ \\
\hline ISSSTE estatal & $12,6 \%$ & $12,3 \%$ & $72,6 \%$ &, $8 \%$ & $1,8 \%$ & $100 \%$ \\
\hline $\begin{array}{l}\text { Pemex, } \\
\text { Defensa o } \\
\text { Marina }\end{array}$ & $17,7 \%$ & $4,7 \%$ & $34,2 \%$ & $1,8 \%$ & $41,5 \%$ & $100 \%$ \\
\hline $\begin{array}{l}\text { Centro de } \\
\text { salud u } \\
\text { Hospital de la } \\
\text { SSA (Seguro } \\
\text { Popular) }\end{array}$ & $27,2 \%$ & $23,3 \%$ & $15,1 \%$ & $20,3 \%$ & $14,1 \%$ & $100 \%$ \\
\hline $\begin{array}{l}\text { IMSS Oportu- } \\
\text { nidades }\end{array}$ & $2,3 \%$ &, $5 \%$ &, $1 \%$ & $45,3 \%$ & $51,8 \%$ & $100 \%$ \\
\hline $\begin{array}{l}\text { Consultorio, } \\
\text { clínica u hos- } \\
\text { pital privado }\end{array}$ & $17,2 \%$ & $22,6 \%$ & $17,6 \%$ & $25,9 \%$ & $16,7 \%$ & $100 \%$ \\
\hline Otro lugar & $23,1 \%$ & $20,0 \%$ & $26,8 \%$ & $16,5 \%$ & $13,6 \%$ & $100 \%$ \\
\hline No se atiende & $28,2 \%$ & $23,7 \%$ & $14,1 \%$ & $22,2 \%$ & $11,8 \%$ & $100 \%$ \\
\hline $\begin{array}{l}\text { No especifi- } \\
\text { cado }\end{array}$ & $39,3 \%$ & $22,5 \%$ & $7,1 \%$ & $22,4 \%$ & $8,6 \%$ & $100 \%$ \\
\hline Totales & $21,4 \%$ & $23,4 \%$ & $16,9 \%$ & $22,5 \%$ & $15,9 \%$ & $100 \%$ \\
\hline
\end{tabular}

Fuente: Elaboración propia con base en el microdato de la base PERS del Censo de Población y Vivienda 2010, INEGI

\subsection{Ingreso de los migrantes que retornaron}

Agrupados los ingresos mensuales en salarios mínimos vigentes en 2010 (1,634 pesos para las cinco entidades), algunas variaciones importantes por entidad son: Guanajuato, Michoacán y Veracruz son las entidades que reportaron más porcentajes de población retornada sin ingresos (entre el 23\% y el 25\% de la población). Mientras Jalisco y Estado de México son las entidades cuya población retornada tienen los mayores porcentajes, 29.7\% el primero y $22.3 \%$ el segundo ganaban más de 5 salarios mínimos (Cuadro 9).

Veracruz (9.5\%), Michoacán (8.9\%) y Guanajuato (7.2\%) son las entidades donde se presenta el mayor porcentaje de migrantes retornados que ganaban hasta un salario mínimo; de un salario hasta 3 salarios mínimos (Guanajuato 54\%, Veracruz 31.2\% y Michoacán 22.9\%). En el caso de Jalisco y Estado de México la población que ganaba más de dos salarios mínimos era $68.7 \%$ y $54.5 \%$ respectivamente (Cuadro 9).

Se puede decir que la desigualdad salarial está relacionada al contexto económico de las entidades federativas como es Jalisco y el Estado de México que tienen una economía más desarrollada; sin embargo, no deber perderse de vista que uno de los problemas principales que ha tenido México desde la década de los ochenta cuando cambia al modelo económico neoliberal, es la pérdida de poder adquisitivo del ingreso. 
Cuadro 9. Ingreso según salario mínimo mensual de los migrantes retornados en 2010

\begin{tabular}{|c|c|c|c|c|c|c|c|c|c|c|c|c|}
\hline \multirow[b]{3}{*}{ Salarios mínimos } & \multicolumn{6}{|c|}{ Dentro de Salarios mínimos } & \multicolumn{6}{|c|}{ dentro de Nombre de la entidad federativa } \\
\hline & \multicolumn{5}{|c|}{ Nombre de la entidad federativa } & \multirow[b]{2}{*}{ Total } & \multicolumn{5}{|c|}{ Nombre de la entidad federativa } & \multirow[b]{2}{*}{ Total } \\
\hline & $\begin{array}{l}\text { o } \\
\frac{0}{0} \\
\frac{2}{\pi} \\
\frac{0}{5} \\
\text { गे }\end{array}$ & $\begin{array}{l}\stackrel{8}{0} \\
\frac{\text { In }}{\mathrm{N}}\end{array}$ & $\frac{8}{\frac{3}{x}}$ & 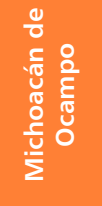 & 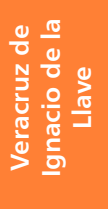 & & 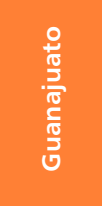 & 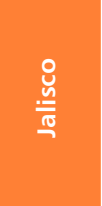 & $\begin{array}{l}\frac{8}{x} \\
\frac{\mathbb{x}}{\Sigma}\end{array}$ & 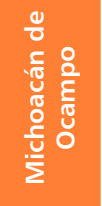 & 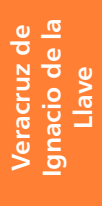 & \\
\hline Sin ingresos & $25,0 \%$ & $16,8 \%$ & $11,2 \%$ & $23,8 \%$ & $23,0 \%$ & $100 \%$ & $13,7 \%$ & $9,4 \%$ & $8,2 \%$ & $13,6 \%$ & $16,2 \%$ & $12,3 \%$ \\
\hline Hasta 1 salario mínimo & $21,4 \%$ & $17,0 \%$ & $13,8 \%$ & $25,7 \%$ & $22,1 \%$ & $100 \%$ & $7,2 \%$ & $5,8 \%$ & $6,2 \%$ & $8,9 \%$ & $9,5 \%$ & $7,5 \%$ \\
\hline $\begin{array}{c}\text { Más } 1 \text { hasta } 2 \text { salarios } \\
\text { mínimos }\end{array}$ & $24,4 \%$ & $15,3 \%$ & $15,3 \%$ & $21,5 \%$ & $23,5 \%$ & $100 \%$ & $25,1 \%$ & $16,1 \%$ & $21,1 \%$ & $22,9 \%$ & $31,2 \%$ & $23,0 \%$ \\
\hline $\begin{array}{c}\text { Más de } 2 \text { hasta } 3 \text { salarios } \\
\text { mínimos }\end{array}$ & $25,2 \%$ & $22,1 \%$ & $17,1 \%$ & $21,9 \%$ & $13,7 \%$ & $100 \%$ & $29,0 \%$ & $26,0 \%$ & $26,3 \%$ & $26,2 \%$ & $20,2 \%$ & $25,7 \%$ \\
\hline $\begin{array}{c}\text { Más de } 3 \text { hasta } 5 \text { salarios } \\
\text { mínimos }\end{array}$ & $18,7 \%$ & $29,8 \%$ & $19,2 \%$ & $21,0 \%$ & $11,3 \%$ & $100 \%$ & $17,0 \%$ & $27,5 \%$ & $23,3 \%$ & $19,7 \%$ & $13,2 \%$ & $20,3 \%$ \\
\hline Más de 5 salarios mínimos & $16,2 \%$ & $29,7 \%$ & $22,3 \%$ & $16,8 \%$ & $15,0 \%$ & $100 \%$ & $8,1 \%$ & $15,2 \%$ & $14,9 \%$ & $8,7 \%$ & $9,7 \%$ & $11,2 \%$ \\
\hline Totales & $22,4 \%$ & $21,9 \%$ & $16,7 \%$ & $21,6 \%$ & $17,4 \%$ & $100 \%$ & $100 \%$ & $100 \%$ & $100 \%$ & $100 \%$ & $100 \%$ & $100 \%$ \\
\hline
\end{tabular}

Fuente: Elaboración propia con base en el micro-dato de la base PERS del Censo de Población y Vivienda 2010, INEGI

\section{Algunas reflexiones}

La migración de retorno considerada como parte del proceso migratorio está estrechamente relacionada a la dinámica de la emigración de mexicanos hacia Estados Unidos. Pero la intensidad y magnitud no es igual en todo el territorio de la República Mexicana, como se señaló previamente, existen procesos y contextos diferenciados que se han analizado por regiones y en algunos entidades federativas de México, coincidimos con López y Mojica (2013) que "el volumen de migración podría representar desafíos de política pública tener algún impacto social, económico, político y en general el reacomodo de la vida cotidiana en las localidades y regiones de la emigración... que deberían de ser atendidas por los tres niveles de gobierno" (López y Mojica 2012, pag 6).

Si bien, la migración de retorno es parte del proceso migratorio se reconoce que el volumen aumentó considerablemente en los últimos años. De acuerdo al Programa Especial de Migración en el periodo de 2005-2010 volvieron al país 1.4 millones de personas, prácticamente el doble que el quinquenio 1995-2000 (García y Zamora 2014, pag 22); sin duda, es relevante conocer el perfil del migrante retornado contemporáneo.
Se ha considerado que el sexo, edad y escolaridad son categorías que permiten identificar la selectividad del migrante. Se podría decir que retornan principalmente hombres, casados en edad productiva, con niveles de escolaridad básica; sin embargo, también están regresando mujeres, niños, jóvenes y ancianos, en algunas localidades en mayor proporción que en otras; por ejemplo, es más claro en el caso de las mujeres que regresan a localidades urbanas.

Entonces la selectividad del migrante de retorno es necesario analizarla con cuidado. Se privilegia la posible vinculación del migrante retorno con el desarrollo de su comunidad o ciudad de origen, pero no sólo depende de las características sociodemográficas y económicas, también es necesario conocer los recursos que ha adquirido en su trayectoria migratoria. Se dice que los migrantes de retorno tienden a insertarse en corto plazo en el mercado laboral, y que más del 70\% consiguen trabajo en tres meses Albo, Ordáz y Li Ng (2012); sin embargo, como se observó la mayoría cuenta con ingresos muy bajos con poca capacidad adquisitiva. De ahí la relevancia de la propuesta de Rivera (2011) de considerar las imbricaciones entre lo local, lo nacional y lo global en la constitución de los sujetos retornados contemporáneos. 
La migración de retorno implica no sólo conocer quienes regresan y a dónde regresan, no es un asunto individual. Si se tiene la capacidad de insertarse a la economía y a la sociedad que lo recibe, sino, también las condiciones sociales, culturales, estructurales que le permitan hacerlo. iSe cuentan con programas de integración o reintegración económica, social y cultural? ¿Existe un sistema de educación y de salud adecuada para la población que retorna? aunque no fue el objetivo del documento responder a estas preguntas, se puede decir que existen retos mayores para que México se prepare para recibir y atender las necesidades del migrante retornado contemporáneo. Porque la migración de retorno es un proceso, la integración o reintegración a la sociedad de origen o de asentamiento presenta diferentes desafíos, para los y las migrantes, para la sociedad de acogida y para el gobierno mexicano.

\section{Bibliografía}

- Albo Marquez Adolfo, Juan Luis Ordaz Díaz, Juan José Li Ng (2012) "Inserción laboral y características de los migrantes mexicanos de retorno 2005-2011. Comparación urbana-rural”. En El estado de la Migración. México ante los recientes desafíos de la migración internacional (Coordinadores) Telésforo Ramírez García y Manuel Ángel Castillo, México, CONAPO.

- Anguiano María Eugenia, Rodolfo Cruz y Rosa María Garbey (2013). "Migración internacional de retorno: trayectorias y reinserción laboral de emigrantes veracruzanos”. En Revista Papeles de Población, No. 77, $\mathrm{pp}$

- Arias, Patricia y Ofelia Woo Morales (2004). "Migración de la zona metropolitana de Guadalajara hacia Estados Unidos". En Revista Papeles de Población. Nueva Epoca, núm 42, año 10, octubre-diciembre, pp. 37-72

- Canales Alejandro I. (2012). "La migración mexicana frente a la crisis económica actual. Crónica de un retorno moderado". En Revista Interdisciplinarda Mobilidade Humana, Brasilia, Ano XX, No. 39, p.117-134, Jul,/dec

- Cassarino, Jean-Pierre. (2004), "Theorising return migration: a revisited conceptual approach to return migrants", In International Journal on Multicultural Societies, June 2004, Vol. 6, No. 2. Italy: EuropeanUniversity Institutehttp://cadmus.eui.eu/bitstream/ id/1906/04_02.pdf/;jsessionid

- Castañeda Camey Nicte Soledad (2014). "El imaginario juvenil urbano sobre la migración y la vida en Estados Unidos”. En Revista Latinoamericana en Ciencias Sociales, Niñez y Juventud, vol 12, número 2, julio-diciembre, pp 617-630

- Cobo Salvador, Silvia Giorguli y Francisco Alba (2010), "La movilidad ocupacional de los migrantes de retorno: un análisis comparativo entre países latinoamericanos". En Salvando fronteras. Migración internacional en América Latina y el Caribe, México,VanderbiltUniverity, MMP, LAMP, Miguel Angel Porrua.

- Durand, J. (2004). Ensayo teórico sobre la migración de retorno. El principio del rendimiento decreciente. Cuadernos geogáficos Num 35 vol. 2, 103-116.

- Durand Jorge y Douglas Massey (2003), Clandestinos. Migración México-Estados Unidos en los albores del siglo XXI. México, Universidad Autónoma de Zacatecas/Miguel Ángel Porrua

- Escobar Latapí, Agustín (2012), "La Política Social Mexicana y Los Migrantes de Retorno” En Empleo Digno, Distribución del Ingreso y Bienestar, coord. Calva, José Luís, (2012). México: Consejo Nacional Universitario y Junan Pablos Editor, pp. 126-153

- Fernández Guzmán, Eduardo (2010), "Migración internacional y retorno migrante contemporáneo”, México: Instituto Mora.

- Fernández Guzmán, Eduardo (2011), Revisión bibliográfica de la migración de retorno, México: Universidad de Guanajuato

- Flores Ávila, Leticia Alma (2010), Hogares urbanos y migración a Estados Unidos. Perspectivas femeninas de tres generaciones de la colonia Constitución, Zona Metropolitana de Guadalajara, 1969-2009. Tesis de 
doctorado en Ciencias Sociales Universidad de Guadalajara.

- García Vega Orlando y Erika Zamora Ramos (2014) "Características Laborales de los Migrantes Mexicanos que regresan a México de Estados Unidos. Un análisis de 1999 a 2013”. En 20 años EMIF, México, CONAPO.

- Gómez Gutiérrez Abel (2014), “iAy Norte, cómo te extraño! tres generaciones de migrantes mexicanos de retorno" en Patricia Galeana (coordinadora) Historia comparada de las migraciones en las Américas. Universidad Autónoma de México e Instituto Panamericano de Geografía e Historia, México pp. 367-392. Disponible en http://biblio.juridicas.unam. $\mathrm{mx} /$ libros/libro.htm?l=3828

- King, Russell (1986) Return migration and regional economic development: an overview. En: King, R. (Ed.) Return migration and regional economic problems). Londres: Croom Helm

- López Castro Gustavo y Mojica Oscar Ariel (2013). "Migración de retorno y los cambios en el índice de intensidad migratoria en Michoacán, Jalisco y Guanajuato”. En Revista Acta Universitaria, Universidad de Guanajuato, Vol. 5, pp 5-15

- Nieto Carlos, 2012. "Migración de retorno y capital humano". En Universitas, enero-julio, Quito, editorial Abya-Yala, pp-53-67

- Papail Jean y Jesús Arroyo, (2004). Los dólares de la migración, Guadalajara, Paris, Los Ángeles, México. Universidad de Guadalajara, Institut De Recherche Pour Le Developpement PROFMEX, Casa Juan Pablos.
- Rivera Sánchez Liliana, (2011). “¿Quiénes son los retornados? Apuntes sobre el migrante retornado en el México contemporáneo”. En La construcción social del sujeto migrante en América Latina. Prácticas, representaciones y categorías Compiladoras Bela Feldman-Bianco, Liliana Rivera Sánchez, Carolina Stefoni y Marta Inés Villa Martínez, CLACSO, FLACSO, Universidad Alberto Hurtado, páginas 309-339 http://webiigg.sociales.uba.ar/pobmigra/ libro_clacso2011.pdf

- Schramm Christian (2011). "Retorno y reinserción de migrantes ecuatorianos, La importancia de las redes sociales transnacionales" En Revista CIDOB d'Afers Internacionals, núm. 93-94, p. 241-260 consultado en www.cidob.org/ca/content/...241-26

- Woo Morales Ofelia (2007). La experiencia migratoria de las mujeres urbanas hacia El Norte. En ¿Campo o ciudad? Nuevos espacios y formas de vida. Coordinadoras Patricia Arias y Ofelia Woo, Universidad de Guadalajara.

\section{Fuentes de Información}

- Consejo Nacional de Población-CONAPO- (2012). Índice Nacional de Intensidad Migratoria México Estados Unidos 2010.ttp://www.conapo.gob.mx/swb/ CONAPO/Indices_de_intensidad_migratoria_Mexico-Estados_Unidos_2010, Consulta en línea mayo 2014.

- Instituto Nacional de Estadística y Geografía -INEGI- (2014). Censo de Población y Vivienda 2010 (microdato de población y vivienda). http://www3. inegi.org.mx/sistemas/microdatos/default2010.aspx Consultada en abril-mayo de 2014 